\title{
A Short Scenario for Restoration of Reasonable Limb Function in Adults after Upper Brachial Plexus Injuries Using Neurotization and Combined Muscle Transfers
}

\author{
MOHAMED A. WAHSH, M.D. \\ The Department of General Surgery, Plastic \& Reconstructive Surgery Unit, Faculty of Medicine, Zagazig University
}

\begin{abstract}
Background: For managing of upper brachial plexus palsy in adult patients, many strategies should be discussed with the patient. Primary plexus repair, nerve transfer, functional muscle transfer and other musculoskeletal approaches may be followed according the suitability, merits and disadvantages of each. Time factorsare very crucial for the sound management for these patients as time delay can result in a non-achievable chance loss with the neuromuscular end plates loss within two years following muscle denervation and progressive musculoskeletal changes as well.

Patients and Methods: Between March 2013 and June 2015, six adult patients suffering from upper brachial plexus injuries were referred to Zagazig University Hospital and operated upon in two stages. In the first stage, two nerve transfers were performed in the same setting; neurotization of suprascapular nerve (using a branch of spinal root of the accessory nerve to the suprascapular nerve) and Oberlin nerve transfer (nerve fascicular transfer from the ulnar nerve to biceps branch of the musculocutaneous nerve to reinnervate the biceps muscle). After three to four months of the first stage, the patients were operated upon in the second stage for combined muscles transfers in the same setting i.e. trapezius transfer to the humerus and latissimus dorsi with teres major transfer to rotator calf of the shoulder.
\end{abstract}

Results: By the end of the tenth month of the first stage, half of patients have regained M4 elbow flexion and the remaining patients have been M3. These improvements have been primarily noticed by the end of the third month postoperatively. The range of elbow flexion has been gradually improved to (100 to 120 degree against resistance) (mean 112 degree) with no noticeable deficit in the hand function as a donor site morbidity. The second stage was done 3 to 4 months from the first stage. Within 6 months of the second stage, shoulder abduction ranged from 80 to 120 degree (mean 98) with the mean of shoulder flexion 77 and externa rotation 47 degrees.

Conclusion: Multimodal association between distal nerves transfer and combined transfer of trabezius, latissimus dorsi and teres major muscles provides an effective and relatively a short management scenario for upper brachial plexus injuries in adult patients.

Key Words: Brachial plexus - Trapezius transfer - Nerve transfer.

\section{INTRODUCTION}

Brachial plexus injury is a considerably disabling problem which has different strategies to deal with. The strategies of management of children and adults' plexus injury differ greatly for many factors. The nerve axons have to travel a considerably shorter distance as well as the regeneration power of the nerve axons is far greater in children [1]. These relative privileges enable extensive plexus reconstruction in children more than adults' palsies in these it may be rarely feasible [2].

In the other hand, physical, psychological and hard nursing supporting care for those adult patients add more difficulties especially for the prolonged periods of seeking medical and surgical management. Unfortunately, this may result in economic and psychological troubles and force some patients to give up seeking the medical care.

Timefactors are crucial for the sound management for these patients. Time delay can result in a non-achievable chance loss as the neuromuscular end plates showed an eventual loss within 20 to 24 months following muscle denervation [3].

Many waiting periods are really subtracted from the remaining time before these permanent changes in the motor end plates within the denervated muscles. This loss affects greatly the scenario of functional improvement.

These periods of delay are such as waiting intervals for management of associated injuries like fractures and post-injury spontaneous recovery periods. Also, patient evaluation, investigations, decision making, availability of suitable facilities, skilled personnel and even periods of ongoing recovery after a trial of primary repair may result in much time loss. 
Functionally, the flexion of the elbow is the main motor function moderator of the upper limb and considered the most precious [4]. The second important function is the abduction of the shoulder as the loss of power to abduct the arm is an incapacitating condition [5].

C5C6 brachial plexus injury generally affects shoulder abduction and elbow flexion causing disabilities and deformities of both the biceps and deltoid muscles. However, if $\mathrm{C} 7$ has been injured with $\mathrm{C} 5,6$ the triceps muscle would have been affected and the wrist extension may be affected as well $[2,6]$. For managing adult upper brachial plexus palsy, many strategies should be discussed with the patients according to their disabilities. Primary plexus repair, nerve transfer, functional muscle transfer and other musculoskeletal approaches may be followed according the suitability, merits and disadvantages of each [2].

In this study we aim to evaluate a relatively short management scenario (totally 6 to 10 months) for adults' upper brachial plexus palsybetween 3 and 15 months of injury. The study used a multimodal management approach as a combination between nerves transfer to the biceps and rotator calf muscles in its first stage surgery followed by multiple muscle transfers in the shoulder in the second stage.

\section{PATIENTS AND METHODS}

Between March 2013 and June 2015, six adult patients, referred to Zagazig University Hospitals, suffering from upper brachial plexus injuries were operated upon in two stages. Those patients (1 women and 5 men) were having a history of injury between 3 and 15 months with no clinical or electroneuromyographic (ENMG) improvement of biceps and deltoid muscles. The first stage may be exclusively done urgently without the initial three months conservation periodfor patients having preganglionic upper plexus injuries diagnosed by imaging (MRI) or ENMG.

In the first stage, two nerve transfers were performed in the same setting:

- Neurotization of suprascapular nerve (using a branch of spinal root of the accessory nerve to the suprascapular nerve).

- Oberlin nerve transfer [4] (transfer of two nerve fascicles from the ulnar nerve to biceps branch of the musculocutaneous nerve to reinnervate the biceps muscle).

After three to four months of the first stage, the following conditions should be fulfilled to continue to the second stage:

- Trapezius strength level should be 5 by MRC scale.

- Complete deltoid paralysis confirmed by electroneuromyography (ENMG).

- Passive shoulder joint abduction should be 80 degree or more.

After fulfillment of these conditions, the patients were operated in the second stage for combined muscles transfers in the same setting:

- Trapezius transfer to the humerus.

- Combined latissimus dorsi transfer with teres major transferto rotator calf of the shoulder.

First Stage
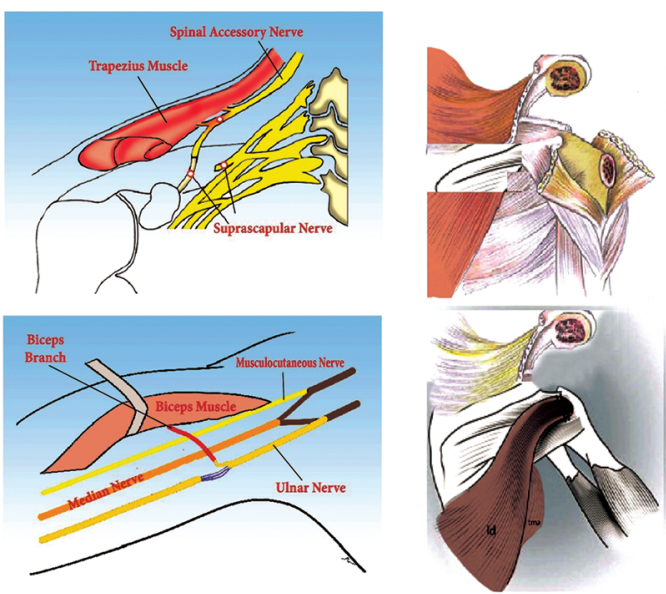

Second Stage

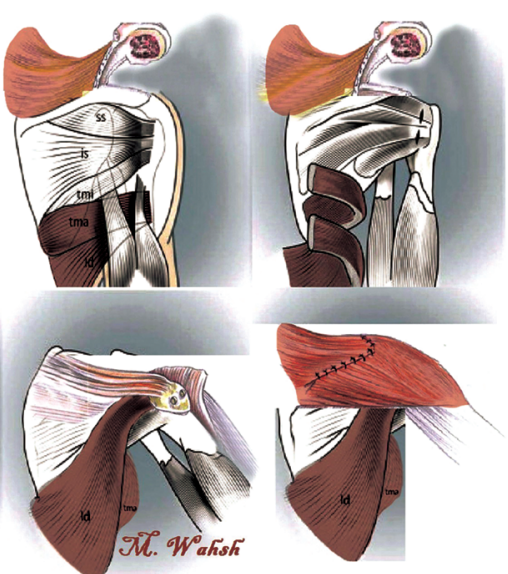

Fig. (1): First stage; suprascapular nerve neurotization by the distal part of the spinal accessory nerve followed by transfer of two nerve fascicles from the ulnar nerve to biceps branch of the musculocutaneous nerve to reinnervate the biceps muscle. Second stage: Elevation of trapezius insertion by division of the acromion base, disinsertion of the tendons of latissimus dorsi and teres major and transfer to the rotator calf and then fixation of the acromion by screws in the humerus. 


\section{Operative Techniques:}

First stage surgery:

The surgeon should clarify to the anesthesiologist to avoid systemic paralytics prior to anesthetic induction to allow proper intraoperative use of the nerve stimulator. Surgical loupes or operating microscope should be used according to the surgeon preference. The patient is put in supine position with his arm abducted 90 degree on an arm Table. A 1/200000 epinephrine solution may be infiltrated in the operative site without any local anesthetic. A supraclavicular transverse incision to skin and dissection of the fascia is made. Under the omohyoid muscle, the suprascapular (the recipient) nerve is identified and taken by a tape to be cut in a healthy segment close to its origin from the upper trunk of the brachial plexus. By following the transverse cervical artery to its entry site in the trapezius muscle, the spinal accessory nerve is searched for at this location, by use of nerve stimulator, which helps to distinguish it from cervical plexus branches. The nerve is then traced distally under the middle and lower trapezius beyond its branches. The large branches of the transverse cervical vessels are carefully divided and the nerve is stimulated again to be reconfirmed and divided as distal as possible. The nerve is then elevated to be anastomosed with the distant cut end of therecipientsuprascpular nerve. Both donor and recipient nerves are anastomosed by fibrin glue and microsutures and closure of the cervical wound. In the upper medial arm, along the bicipital groove, other incision is done with dissection through subcutaneous tissue and fascia. The medial antebrachial nerve should be identified and protected. The median nerve is centered on the brachial artery and the ulnar nerve is identified medially. The biceps muscle is elevated laterally to follow the musculocutaneous nerve proximally and its branches. The musculocutaneous has three branches; a branch to the biceps (BBi) courses laterally and separates from the musculocutaneous nerve proximally and another branchto the brachialis (BBr) courses medially and lateral antebrachial nerve continues distally. Firstly, we test these branches using the nerve stimulator to confirm the absence of any muscle activity. The biceps branch is dissected proximally allowing more length for the anastomosis as proximal as possible (the recipient). The ulnar nerve is dissected more distally with opening of its epineurium and separation of a group of fascicles by a fine, microscopic technique. By using the nerve stimulator, we select two fascicles (the donor) with pure wrist flexion action that often located on the lateral or central fascicles. The donor and recipient fascicles are dissected properly and cut after making sure that the anastomosis would not be under tension. The two ends are coaptated and anastomosed by fibrin glue and micro-sutures. The wound is closed in layers with soft drain and wrapped with bulky gently compressive dressing. Then, the limb is put in an arm sling for 3 weeks. Re-education and electrotherapy is done from the endof the second month till the second stage surgery.

\section{Second stage surgery:}

The patient is placed in a lateral position and a skin incision along with the anterior, lateral, and posterior deltoid origin then continue posteriorly on the back of the shoulderthen along with the posterior axillary fold. Elevation of the insertion of the trapezius by dividing the root of acromion and the lateral end of the clavicle lateral to the coracoclavicular ligaments, using a Gegli saw, with release of the remaining insertion of the trapezius from the clavicle and the spine of the scapula. The proximal humerus is exposed by undermining the deltoid. Latissimus dorsi and teres major tendons are disinserted from the humerus with putting the humerus in 90 degree of abduction and full external rotation. This position should be kept until the limb be put in an airplane splint immediately postoperatively. In this position, latissimus dorsi tendon is fixed to rotator calf (two non-absorbable sutures at least or by using an anchor fixator to the humerusbone to obtain a reliable fixation) and teres major muscle is rerouted and reattached to the latissimus dorsi tendon with an appropriate extra-articular musculotendinous lengthening. The acromial fragment with its insertion of trapezius is then transferred and fixed by two $4.5 \mathrm{~mm}$ screws to the humerus after roughening the attachment by an osteotome. The fixation is done with the arm in 90 degree arm abduction and full external rotation.

A radiograph is then taken to check the proper fixation position followed by suturing the deltoid over the trapezius as proximal as possible, to enforce the trapezius transfer and the potential deltoid reinnervation recovery if any. The skin isthen closed with a suction drain. After operation, the arm is immobilized at $80^{\circ}$ of abduction and full external rotation in an airplane splint for 10 weeks with gradual weaning of the splint for another two weeks then gradual therapy is started (Fig. 4).

The patients were evaluated for shoulder joint stability i.e. clinically and radiologically, shoulder pain, back pain, scoliosis and gait changes (Table $1)$. 
The patients' results were reported just before the first stage, before the second stage i.e. about
3 months after the first stage, 3 months after the second stage and 6 months after the second stage.
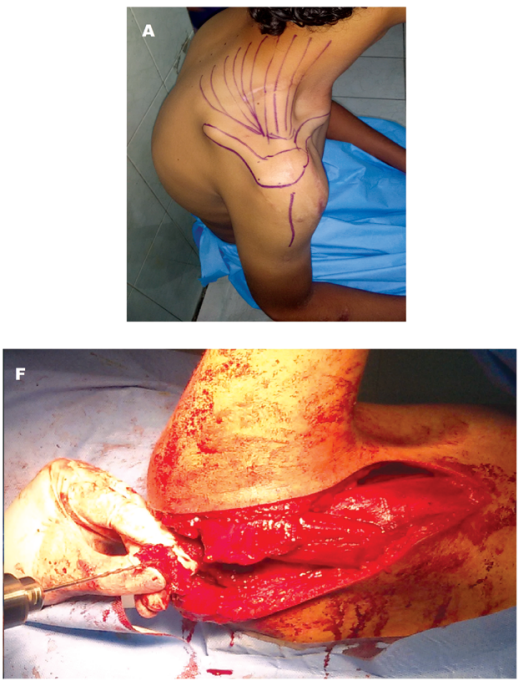
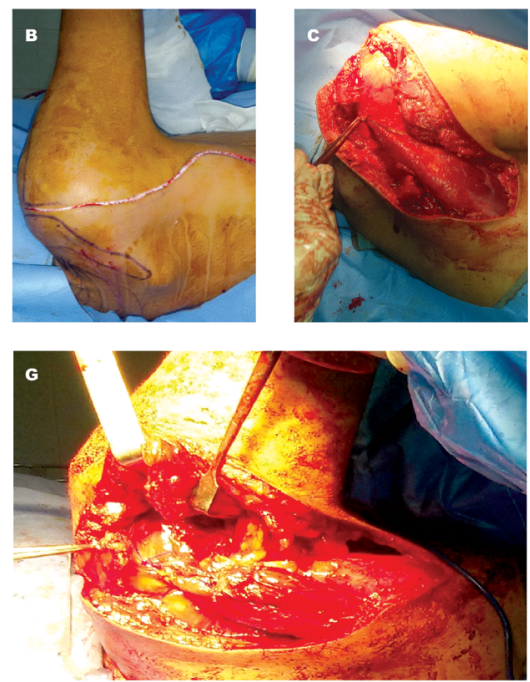
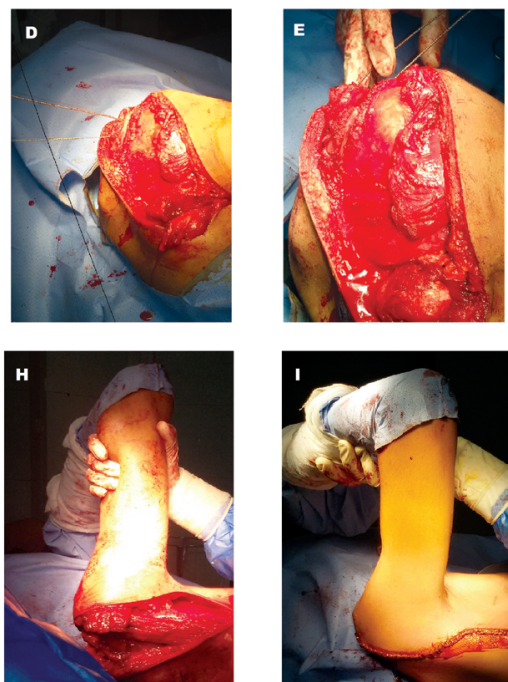

Fig. (2): Operative technique of the trapezius, latissimus dorsi and teres major transfer. A- Preoperative marking. B- Skin incision along with the anterior, lateral deltoid origin, back of the shoulder and posterior axillary fold. C,D,E. division of the origin of the deltoid from acromion, disinsertion of L.D. and T.M from the humerus and bone division of the acromion base and lat. clavicle. F,G,H,I. transfer of the L.D. to rotator. calf, T.M. to L.D. tendon, acroion fixation by screws to tumerus and closure.

\section{RESULTS}

Six patients were studied in the current study (5 males and one female) with ages ranging from 18 to 42 years. The time intervals between injury and surgery were from 3 to 15 months.

Shoulder joint instability were detected as early as 5 months after injury however shoulder pain was reported in half number of patients of the study. Scoliosis and gait changes were present in all patients included in the study.

In the six patients, as shown in the Tables $(2,3)$, four types of movementshave been evaluated; shoulder abduction, external rotation, shoulder flexion and elbow flexion according to the angle of the movement against gravity. Furthermore, according to Medical Research Council scale (MRC), elbow flexion was evaluated. MRC scale is amuscle power scoring system ranging from 0 to 5 ; 0 no contraction, 1 flicker or trace of contraction, 2 active movement with gravity eliminated, 3 active movement against gravity, 4 active movement against gravity and resistance and 5 normal power. During evaluation of the external rotation of the shoulder, the arm is hanged in 90degree abduction with the elbow is put in 90 degree flexion (neutral position) and the patient is asked to rotate the arm by elevating the forearm from the horizon and the degree of the external rotation is evaluated by the forearm elevation. Also, the wrist and hand muscles were evaluated before and after.

The first and second stages and the trapezius power were mandatory confirmed to have a normal power (MRC5) and the passive abduction of the shoulder was exceeding 80 degree as a preliminary condition for the second stage.

The six patients were operated for Obrelin procedure by using 2 ulnar nerve fascicles transferred to the biceps muscle. By the end of the tenth post-operative month, half of patients have regained M4 elbow flexion and the remaining patients have been M3. These improvements have been primarily noticed by the end of the third month postoperatively. The range of elbow flexion has increased throughout the follow up visits to reach 120 degree against gravity (mean 112 degree) with no noticeable deficit in the hand function as a donor site morbidity. (Table 2) (Fig. 3).

After the second stage, the patients were put in airplane splint for ten weeks complete splinting and two weeks of gradual weaning. Within six months from the second stage, shoulder abduction ranged from 80 to 120 degree (mean 98 degree) with the forward shoulder flexion was ranging from 50 to 90 degree (mean 77 degree) and external rotation was ranging from 10 to 70 degree (mean 47 degree). (Table 3) (Fig. 4). 

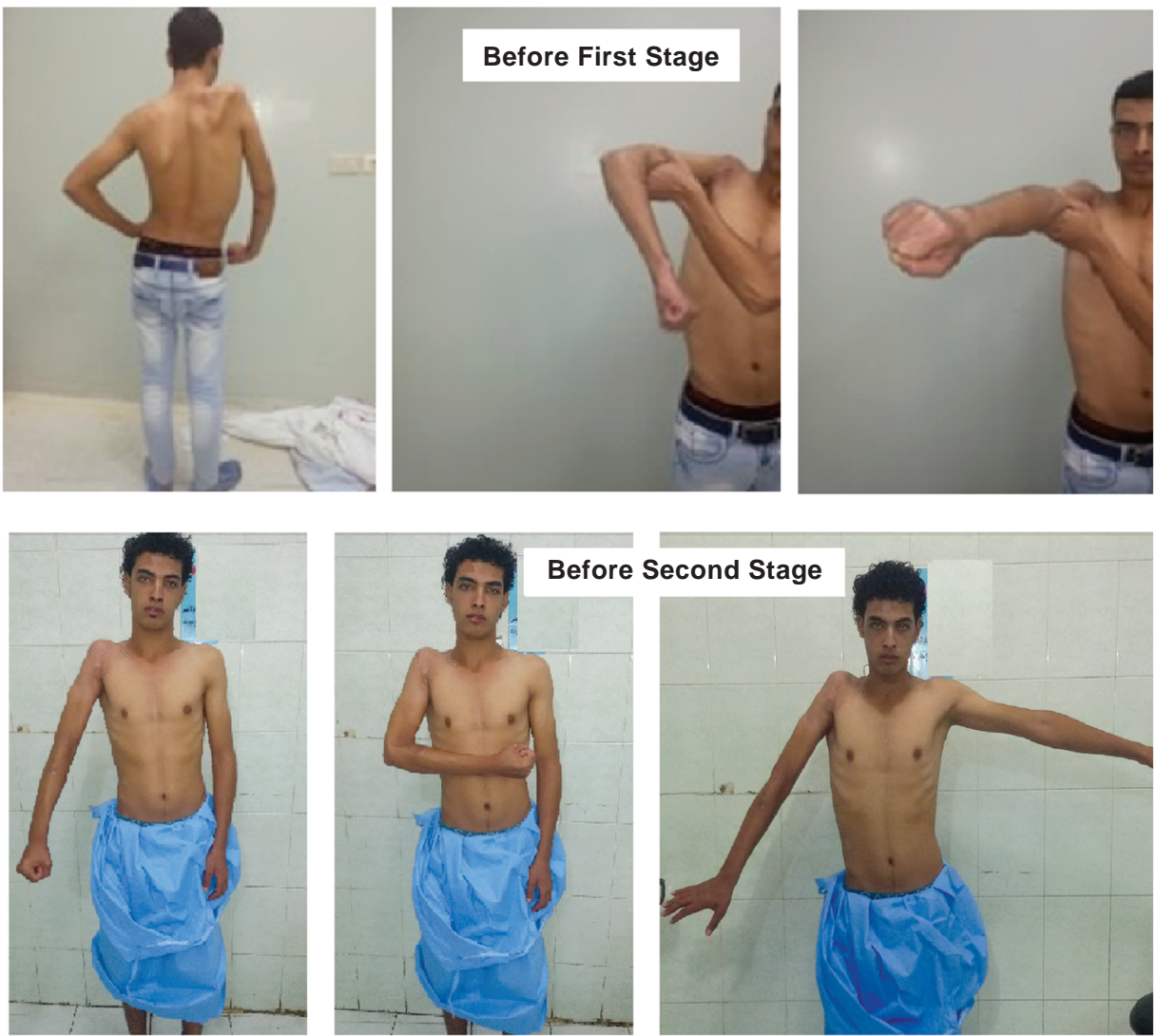

Fig. (3): Pre-operative views of the first stage surgery with complete paralysis of shoulder abduction and elbow flexion. Preoperative views of the second stage surgery with improvement of the elbow flexion $\left(100^{\circ}\right)$ and partial improvement of the right shoulder abduction (reinnervation of the biceps and supraspinatus muscles in the first stage).
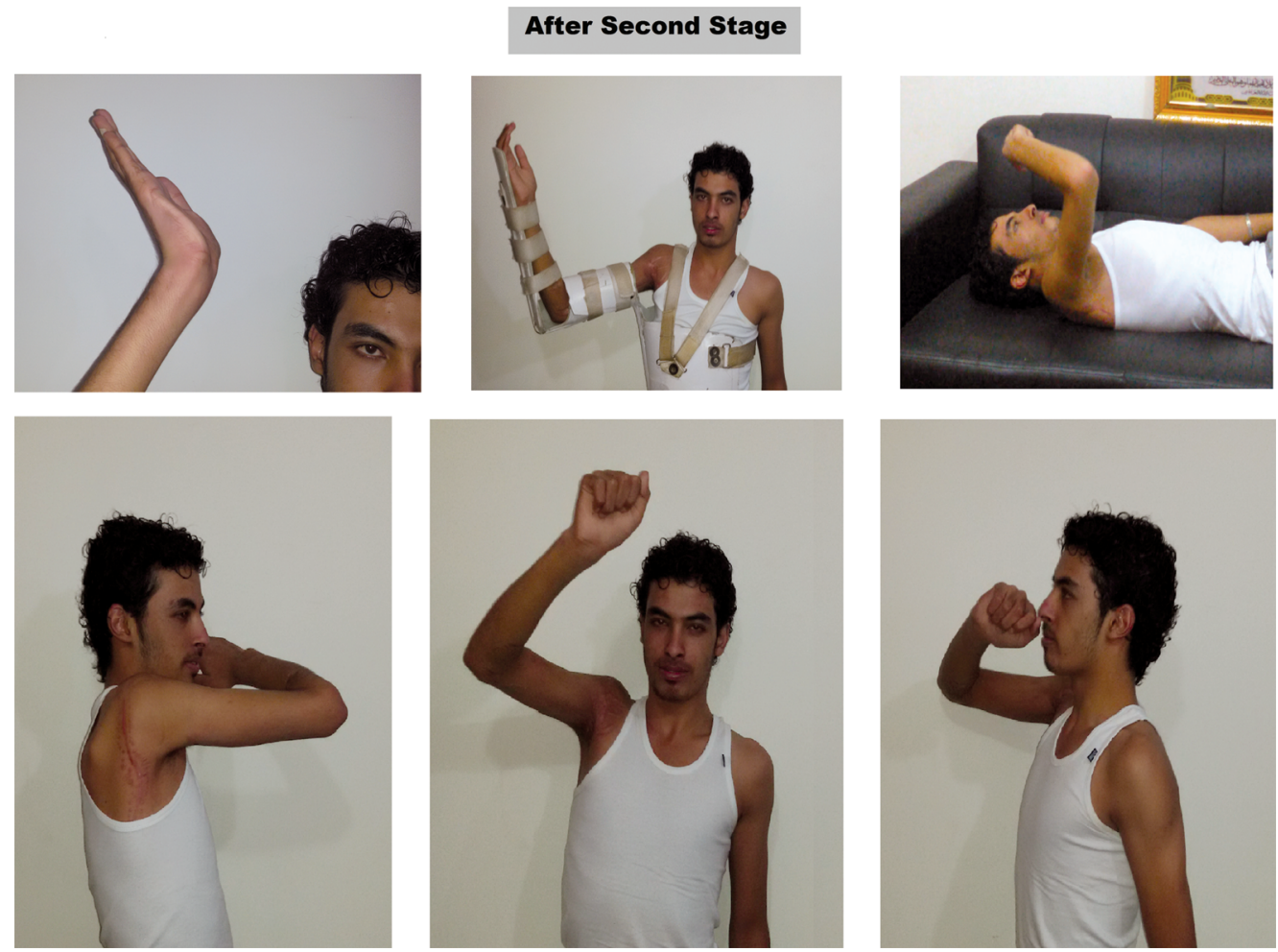

Fig. (4): Post-operative views of the second stage surgery with improvement of the shoulder abduction $\left(110^{\circ}\right)$, flexion $\left(90^{\circ}\right)$ and external rotation $\left(50^{\circ}\right)$ in addition to more improving of the elbow flexion. The hand has not been affected by ulnar fascicles donation (no fingers clawing). The splinting of the limb in the airplane splint and gradual exercises for weaning from splint are also shown. 


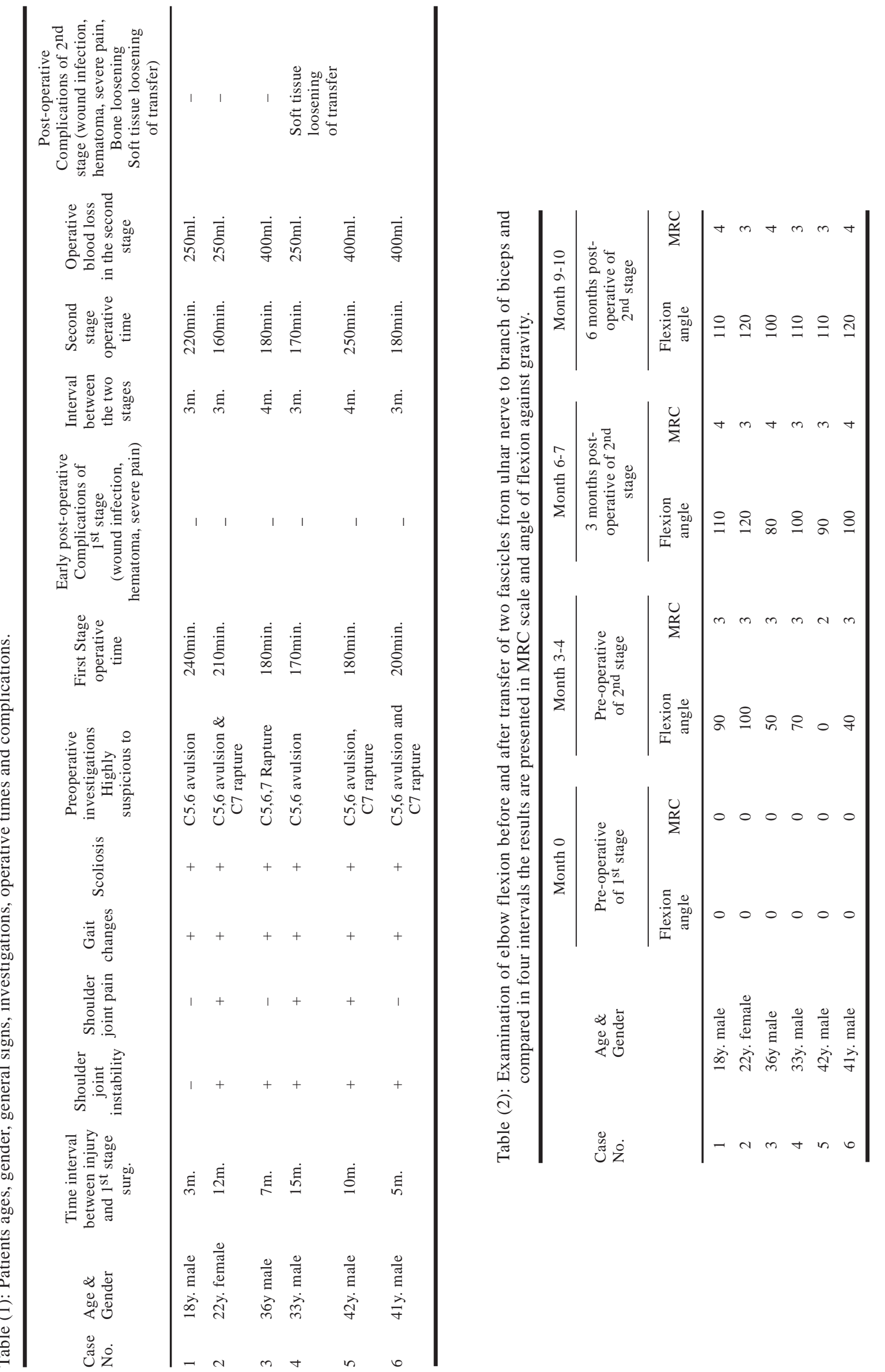




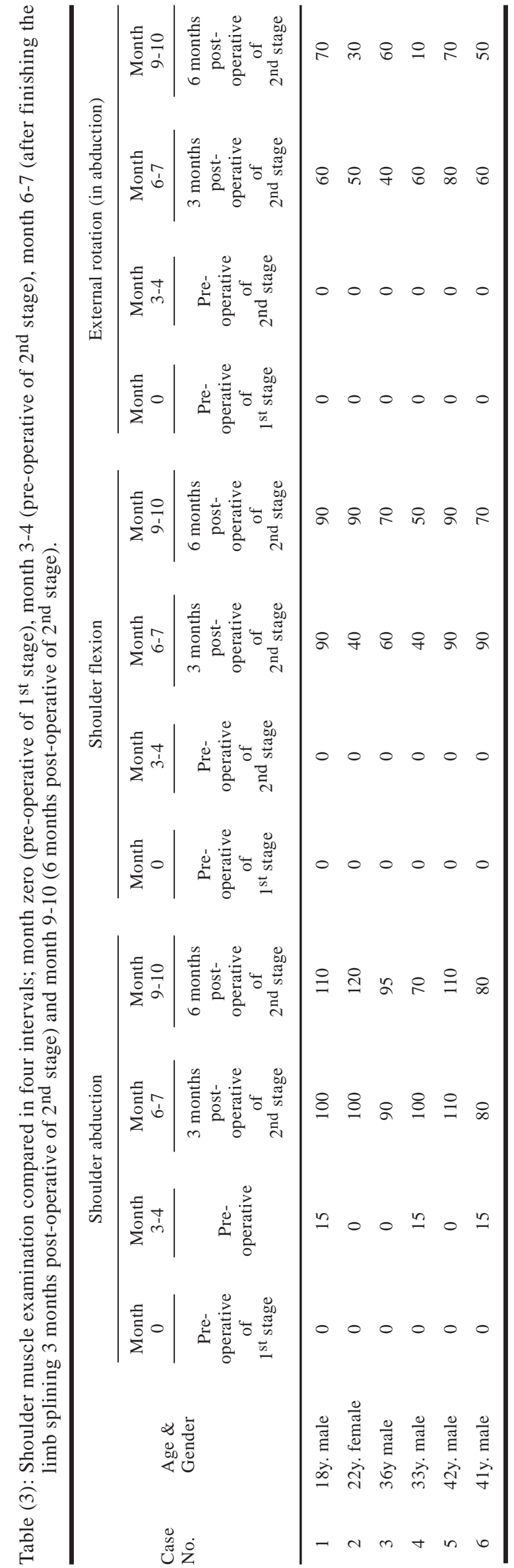

\section{DISCUSSION}

Functionally, the flexion of the elbow is the main motor function of the upper limb and considered the most precious [4]. The second important function is the abduction of the shoulder and the loss of power of the arm abduction is an incapacitating condition [5].

C5C6 injury generally affects shoulder abduction and elbow flexion causing marked disabilities and deformities of both the biceps and deltoid [6]. However, if $\mathrm{C} 7$ has been injured with $\mathrm{C} 5,6$ the triceps muscle would have been affected and the wrist extension may be affected as well $[\mathbf{2 , 6 ]}$.

By target-based analysis of thesedisabilities, the primary goal in managing their cases is the combined regaining of both elbow flexion, shoulder stability and shoulder abduction otherwise the results would be considered by the patient as unfavorable. Also, other secondary goals are necessary to achieve like shorting themanagement time and regaining adequate shoulder external rotation.

For many surgeons, whenever the time is feasible, the primary nerve repair is considered to have the most priority over the other options in order to deal with the injuries primarily wheneverfeasible asearly as possible [2]. Unfortunately, this statement is not the rule in adult brachial plexus palsies and traditional brachial plexus exploration and reconstruction may result in poor functional outcomes [6].

Oberlin's used a nerve transfer from ulnar nerve to biceps muscle in 1994 as an alternative way to regain the biceps function [4]. Leechavengvongs in 1998 reported restoration of biceps function and elbow flexion MRC power M3 to M4 with the same technique [7]. Furthermore, Mackinnon's added other fascicular neurotization of the brachialis muscle from the ulnar nerve with even better results $[8,9]$. Currently, the transfers performed by those surgeons are gaining more popularity as their works have created an exciting new strategy in management of brachial plexus palsy [2]. This strategy has carried out much quick reinnervation with higher chances of success $[4,10]$.

In the current study, 6 patients were operated upon using Obrelin procedure by transferring two ulnar nerve fascicles selected by a nerve stimulator. These fascicles usually have beentaken from the fascicles supplying the wrist flexors and transferred to the biceps muscle. In the third post-operative month the flickers or good flexion power have 
been primarily noticed. By the end of the tenth month, half of patients have regained M4 elbow flexion and the remaining patients have been M3. Furthermore, the range of elbow flexion has increased throughout the follow-up visits to reach 120 degree against gravity and mild resistance (mean 112 degree) with no noticeable deficit in the hand function as a donor site morbidity. Sungpet [11] reported the most favorable results by obtaining MRC 3 or more within 3.3 months in thirty four out of thirty-six patients when he innervated the biceps by a single fascicle of the ulnar nerve. Also, the hand function of this series was not compromised through a prolonged follow-up period [11].

The present study assumes that waiting for long graft repair in upper brachial plexus injuries for restoration the shoulder abduction is not the best choice. In consistent to our assumption, somsak [12] invented a nerve transfer to the deltoid from the nerve of the long head of the triceps muscle. However, although this is a useful addition for the management of the brachial plexus injuries it is not suitable for delayed cases or injured $\mathrm{C} 7$ asdonor branchwould be non-functioning. Also, it would improve the deltoid function only with no effect on external rotation.

Although, secondary procedures are generally usedafter full neurological management and adequate physiotherapy $[\mathbf{2 , 1 3 , 1 4 , 1 5 ]}$. In this study, only regarding the shoulder joint, we assume that it is not an evidence base attitude. Muscle transfer is relatively simple techniques with high incidence of success and comparable functional results. Trapezius, rhomboid muscles and levator scapulae, in $96 \%$ of cases, are usually healthy and available for transfer [16]. Aziz, Singer and Wolff [17] when studied trapezius transfer after brachial plexus palsy, reported that it is a simple procedure with functional improvement regarding the shoulder abduction, pain elimination, joint stability and can be performed in combination with other muscles transfer to maximize the limb function [16,17]. Also, he argues that trapezius transfer was compatible with any progression of the function of the other shoulder muscles [17].

In the current study, we used in the first stage reinnervation of suprascapular nerve of the infraspinatus and supraspinatus muscles nerve in association with Oberlin neurotization of biceps muscle. The second stage a combined muscle transfer (trapezius muscle to the humerus plus latissimus dorsi to rotator calf and teres major muscle augmenting this muscle function). By combination between the two stages we could combine two fast track methods.

In the present study, by combining muscles transfer, shoulder abduction ranged from 80 to 120 degree (mean 98 degree) with the mean of shoulder flexion 77 degree and external rotation 47 degree after six months follow-up period.

In consistent to the current study, Gilbert et al., [18] concluded that trapezius transfer and latissimus dorsi rerouting resulted in good results. However, Saha [24] confirmed that when transfer of a single muscle to replace the deltoid the angle of abduction did not exceed $90^{\circ}$ Chen et al., [19] and Terzis and Kokkalis [20]. combinedlatissimus dorsi andteres major transfer to the rotator calf muscles with the trapezius muscle transfer with significant global shoulder function improvement. Average abduction of 120 degree was reported by Narakas [21]. with trapezius transfer with latissimus dorsi, teres major and levator scapulae. Also, Karev [22] obtained a full range of movementin a patient by the same technique. Chun-lin and Yong-hua [23] reported seven cases of pectoralis major transfers with additional trapezius transfer.

Conclusion: Multimodal association between distal nerves transfer followed by combined transfer of trapezius, latissimus dorsi and teres major muscles provides an effective and relatively short management scenario for upper brachial plexus injuries in adult patients.

\section{REFERENCES}

1- Thatte M.R. and Mehta R.: Obstetric brachial plexus injury. Indian J. Plast. Surg., 44: 380-9, 2011.

2- Thatte M.R., Babhulkar S. and Hiremath A.: Brachial plexus injury in adults: Diagnosis and surgical treatment strategies. Ann. Indian. Acad. Neurol. Jan-Mar., 16 (1): 26-33, 2013.

3- Ferrante M.A.: Electrodiagnostic Assessment of the Brachial Plexus. Neurol. Clin., 30: 551-80, 2012.

4- Oberlin C., Beal D., Leechavengvongs S., Salon A., Dauge M.C. and Sarcy J.J.: Nerve transfer to biceps muscle using a part of ulnar nerve for C5-C6 avulsion of the brachial plexus: Anatomical study and report of four cases. J. Hand Surg., 19A: 232-237, 1994.

5- Siqueira M.G. and Martins R.S.: Surgical treatment of adult traumatic brachial plexus injuries: An overview. Arq. Neuro-Psiquiatr. Vol. 69, No. 3, São Paulo June 2011.

6- Nerve Transfer for Restoration of Elbow Flexion in Upper Brachial Plexus Injuries. Orthopaedics One Articles. In: Orthopaedics One - The Orthopaedic Knowledge Network. Created Sep., 19: 09-04. Last modified Oct., 06, 14- 20 ver. 7, 2011. 
7- Leechavengvongs S., Witoonchart K., Uerpairojkit C., Thuvasethakul P. and Ketmalasiri W.: Nerve transfer to biceps muscle using a part of the ulnar nerve in brachial plexus injury (upper arm type): A report of 32 cases. J. Hand Surg., 23A: 711-6, 1998.

8- Humphreys D.B. and Mackinnon S.E.: Nerve transfers. Op. Tech. Plast. Reconstr. Surg., 9: 89-99, 2002.

9- Tung T.H., Novak C.B. and Mackinnon S.E.: Nerve transfers to the biceps and brachialis branches to improve elbow flexion strength after brachial plexus injuries. J. Neurosurg., 98: 313-318, 2003.

10- Mackinnon S.E. and Dellon A.L.: New York: Thieme Medical Publishers Surgery of the Peripheral Nerve., 1988.

11- Sungpet A., Suphachatwong C., Kawinwonggowit V. and Patradul A.: Transfer of a single fascicle from the ulnar nerve to the biceps muscle after avulsions of upper roots of the brachial plexus. J. Hand Surg. [Br.], 25: 325-328, 2000 .

12- Leechavengvongs S., Witoonchart K., Uerpairojkit C. and Thuvasethakul P.: Nerve transfer to deltoid muscle using the nerve to the long head of the triceps, part II: A report of 7 cases. J. Hand. Surg. Am., 28: 633-8, 2003.

13- Berger A. and Becker M.H.: Brachial plexus surgery: Our concept of the last twelve years. Microsurgery, 15: 7607, 1994.

14- Goldner J.L.: Strengthening of the partially paralyzed shoulder girdle by multiple muscle-tendon transfers. Hand Clin., 4: 323-36, 1988.

15- Jäger M. and Wirth C.J.: Shoulder arthrodesis and muscle transplantation for the functional improvement of upper- plexus lesions. Aktuelle Probl. Chir. Orthop., 1: 80-3, 1977.

16- Monreal R., Paredes L., Diaz H., et al.: Trapezius transfer to treat flail shoulder after brachial plexus palsy. J. Brachial. Plex. Peripher. Nerve Inj., 2: 2, 2007.

17- Aziz W., Singer R.M. and Wolff T.W.: Transfer of the trapezius for flail shoulder after brachial plexus injury. J. Bone. Joint. Surgery. (Br.), 72-B: 701-4, 1990.

18- Gilbert A., Brockman R. and Carlioz H.: Surgical treatment of brachial plexus birth palsy, Clin. Orthop., 264: 39-47, 1991.

19- Chen L., Gu Y.D. and Hu S.N.: Applying transfer of trapezius and/or latissimus dorsi with teres major for reconstruction of abduction and external rotation of theshoulder in obstetrical brachial plexus palsy. J. Reconstr.

20- Julia K. Terzis and Zinon T.: Kokkalis Orthoplasty in Trauma and Reconstructive Microsurgery: Primary and secondary shoulder reconstruction in obstetric brachial plexus palsy Injury, 39 (3): 5-14, 2008.

21- Narakas A.O.: Muscle transpositions in the shoulder and upper arm for sequelae of brachial plexus palsy. Clin. Neurol. Neurosurg., 95: Suppl. 89-91, 1993.

22- Karev A.: Trapezius transfer for paralysis of the deltoid. J. Hand Surg. [Br.], 11-B: 81-3, 1986.

23 Chun-lin H., Yong-hua T.: Transfer of upper pectoralis major flap for functional reconstruction of deltoid muscle. Chin. Med. J., 104: 753-7, 1991.

24- Saha A.K.: Surgery of the paralyzed and flail shoulder. Acta. Orthop. Scand, Suppl. 97, 1967. 